\title{
A Semi-Analytical Approach for Performance Evaluation of RTD-based Oscillators
}

\author{
Rafael Nobrega ${ }^{1,2}$, Ulysses Duarte ${ }^{2}$, Thiago Raddo ${ }^{3}$, Ivan Glesk ${ }^{4}$, Anderson Sanches ${ }^{1,4}$, and Murilo Loiola ${ }^{1}$ \\ ${ }^{1}$ Engineering, Modeling, and Applied Social Sciences Center, Federal University of ABC, Santo André, Brazil \\ ${ }^{2}$ Academic Area of Electrical Engineering, Federal Institute of Minas Gerais, Formiga, Brazil \\ ${ }^{3}$ Institute for Photonic Integration, Eindhoven University of Technology, Eindhoven, The Netherlands \\ ${ }^{4}$ Faculty of Engineering, University of Strathclyde, Glasgow, United Kingdom \\ Tel: (5511) 4996 8289, e-mail: rafael.nobrega@ufabc.edu.br
}

\begin{abstract}
In this paper, it is presented a comprehensive analysis of three optoelectronic oscillators circuits based on resonanttunneling diodes (RTD) for promising applications in terahertz frequency (THz) range. For all cases, it is investigated how each of these RTD devices performs under a wide range of the direct current (DC) voltages within the negative differential resistance region (NDR). The mathematical formalism developed here accounts for the effect of bias applied on the parameters of the reactive elements that composed the oscillators' small signal model. In this context, new expressions for evaluating the width of the depletion region of the devices are derived. Such mathematical formalism allows to make the performance analysis straightforward and show a close agreement to the device characterizations presented in the literature. Results pave the way for these RTD-based oscillators as potential candidates for Radio-over-Fiber (RoF) transmission, where higher operation frequencies will be major network requirements.
\end{abstract}

Keywords: resonant tunneling diode, oscillator circuit, terahertz applications, radio-over-fiber.

\section{INTRODUCTION}

In the past few years, there has been an increasing demand for broadband services offered by wireless networks. This can be attributed to users experience in potential multimedia applications such as video on demand, high definition television (HDTV), internet protocol television (IPTV) and high internet speed. In this context, a great deal of research has been made to developing efficient approaches which increase data traffic significantly. As it is well known, such growth of traffic patterns can only be attended by a substantial improvement in the signal-to-noise ratio or the increase in the available bandwidth for transmission. The first alternative is impracticable since it has high transmission powers requirements for a given bit error rate (BER). The second alternative, on the other hand, is entirely feasible if considered the move to the unfilled terahertz frequencies (THz) region with large bandwidth [1]-[6]. Nonetheless, even though a great deal of effort was dedicated to the development of efficient approaches to manipulate signals at such spectrum, technological limitations made it impossible to circumvent the attenuation experienced by waves oscillating in $\mathrm{THz}$ frequencies in free space. Fortunately, the overall reach of the network can also be significantly improved with the use of emerging technologies based on Radio-over-Fiber (RoF). Further, the overall performance of the system can also be substantially improved if, besides using RoF networks, one even implements optoelectronic circuits based on resonant tunneling diode (RTD) at the transmitter side [6], [8]. In this case, intrinsic characteristics of RTDs allow to convert from wireless to optical domains exploring some remarkable aspects of this device, such as fundamental oscillation in highest frequency, fast switching times, inherent negative differential conductance characteristics at the low applied voltage, high compatibility with conventional device technologies, and room temperature operation [6]-[9]. However, there is a lack of modeling for the behavior of RTD oscillators concerning quantitative analysis of their related parameters operating at $\mathrm{THz}$ frequencies. Furthermore, many approaches available in the literature leads to enormous complications to solve its mathematical formalisms and then infer the oscillators' parameters [5]. Based on the earlier discussion, one might see that there are still some aspects of RTDs modelling that are yet to be accounted for. For example, a model that adequately includes not only the RTD reactive elements dependence over their intrinsic parameters but also have a straightforward solution.

In this context, this paper presents for the first time, as far as the authors are aware of, a comprehensive analysis of three distinct RTD oscillators. For all cases, it is derived from new expressions for evaluate the width of the depletion region and investigated how the direct current (DC) voltage performs on the oscillation frequency, maximum frequency and extinction ratio of the RTD devices, which are the figure of merits adopted in this analysis.

\section{MATHEMATICAL FORMALISM}

In this section, it is developing the mathematical framework that will be utilized to model the oscillators based on the RTD devices. The formalism incorporates fitting parameters extracted from the experimental current versus voltage characteristics (I-V) to further evaluate the width of the depletion region of the devices in a semi-analytical approach. Besides, such semi-analytical approach assumes that the potential drop behavior after the second barrier takes on the 
conventional diode pattern. This assumption can be supported by input voltages within the NDR and allow greatly simplify the mathematical formalism.

The proposed formalism explicitly requires the I-V characteristics to be defined in terms of fitting parameters. A straightforward expression that gives an excellent fit to the experimental data is provided by [1]

$$
I(V)=\operatorname{Area}\left\{A \ln \left[\frac{1+\exp \left[\frac{\left(B-C+n_{1} V\right)}{V_{t h}}\right]}{1+\exp \left[\frac{\left(B-C-n_{1} V\right)}{V_{t h}}\right]}\right]\left(\frac{\pi}{2}+\tan ^{-1}\left(\frac{C-n_{1} V}{D}\right)\right)+H\left(\exp \left(\frac{n_{2} V}{V_{t h}}\right)-1\right)\right\}
$$

where Area is the cross-sectional area of the device, $A, B, C, D, n_{1}$ and $n_{2}$ are fitting parameters, $V_{t h}=k B T / q$ is the thermal voltage, $k_{B}$ is the Boltzmann constant, $q$ is the elementary charge and $T$ is the operating temperature.

In addition, it has been usual in the literature to model the RTD as a parallel-inductance equivalent circuit, which the inductance appears in series with the tunneling conductance and the total is in parallel with the junction capacitance. In this case, the quantum inductance originating from the lagging property of the quantum well charge with bias change is written as [2]

$$
L(V)=\frac{\tau}{G(V)},
$$

where $\tau$ is the tunneling time [3] and $G(V)=d I(V) / d V$ is the differential conductance. It is worth mentioning that $\tau$ is a function of the intrinsic parameters and the energy level of the first resonance state inside the quantum well. In all simulations, the values of such a resonant state were obtained via the Transfer-Matrix Method (TMM) [4].

The total RTD capacitance including both the geometrical depletion capacitance and the quantum capacitance are given, on the other hand, can be expressed by [2]

$$
C(V)=-\tau_{c} G(V)+\frac{\text { Area }}{\frac{L_{w}}{\varepsilon_{w}}+2 \frac{L_{b}}{\varepsilon_{b}}+\frac{L_{d}(V)}{\varepsilon_{d}}},
$$

where $\tau_{c}$ is the scape rate through the collector barrier [3], $L_{w}$ and $\varepsilon_{w}$ are the width and the permittivity of the quantum well, respectively, $L_{b}$ and $\varepsilon_{w b}$ are the width and the permittivity of the barrier, respectively, $L_{d}$ and $\varepsilon_{d}$ are the width and the permittivity of the depletion region, respectively.

Since all the relevant calculation parameters for the earlier expression have appropriately been described, the width of the depletion region can now be discussed. In this context, the expression for the width of the depletion region can be adapted from [1] based on the specific features of the here proposed approach, where oscillators achieve an operating frequency of the order of THz. Now, considering that the characterization of the RTD device is known the width of the depletion region is given by

$$
L_{d}=\left[\frac{2 \varepsilon_{d} V_{i n}}{q}\left(\frac{1}{N_{a}}+\frac{1}{N_{d}}\right)\right]^{M},
$$

where $N_{a}$ and $N_{d}$ are the acceptor and donor concentrations in the collector region, respectively, and $M$ is an empiric factor estimated from the characteristic curves of the devices. Full details on the proposed mathematical framework will be published elsewhere.

Next, once all pertinent reactive elements have been accounted for, it is possible to introduce the values of $R_{s}$ to meet the stability criteria of the oscillator circuit [5]. Then, the merit figures named oscillation frequency, maximum frequency and extinction ratio were obtained by numerically solving the time-dependent first-order differential equation based on voltage and current (Van der Pol equation) [5].

Finally, the maximum frequency (estimated by calculating the resistive cutoff frequency of the small-signal equivalent circuit model) of the RTD-based oscillator circuit can be expressed as [6],

$$
f_{\max }=\frac{1}{2 \pi C} \sqrt{\frac{-G_{\max }}{R_{s}}-G_{\max }^{2}}
$$

\section{RESULTS AND DISCUSSION}

The semi-analytical formalism developed in the previous section is now applied to evaluate the performance of three RTD-based oscillators. In all situations, the values of the reactive elements that composed the oscillators' small signal model are obtained from the expression for the width of the depletion region proposed here. Nonetheless, before proceeding with the performance analysis itself, it is interesting to verify the accuracy of the formalism proposed here. It is noteworthy that here-proposed formalism converges to experimental results when the voltage inputs are within the NDR region of RTD devices. In order to prove the above statement, Table I shows a comparison of the depletion regions widths obtained through the proposed formalism with four works previously reported in the literature. The close agreement between the previous results and those obtained with the here-proposed mathematical framework proves the ability of this formalism to correctly evaluate the values of depletion regions widths of RTD devices. 


\begin{tabular}{|l|l|c|c|c|c|c|}
\hline Ref. & Process & $N_{a}\left(\mathrm{~cm}^{-3}\right)$ & $N_{d}\left(\mathrm{~cm}^{-3}\right)$ & $V_{i n}(V)$ & $L_{d}(\mathrm{~nm})$ & $\begin{array}{c}\boldsymbol{L}_{\boldsymbol{d}, \text { sim }}(\boldsymbol{n m}) \\
\text { This Work }\end{array}$ \\
\hline$[6]$ & GaN/AlN & $1.5 \times 10^{15}$ & $10^{17}$ & 9.7 & 36 & 35.5 \\
\hline$[7]$ & GaAs (wafer 1) & $3 \times 10^{14}$ & $10^{18}$ & 0.4 & 15 & 14.9 \\
\hline$[7]$ & GaAs/Al $0.3 \mathrm{Ga}_{0.7} \mathrm{As}$ (wafer 2) & $0.9 \times 10^{14}$ & $2 \times 10^{17}$ & 0.32 & 30 & 30.1 \\
\hline$[7]$ & GaAs (wafer 3) & $0.7 \times 10^{14}$ & $2 \times 10^{17}$ & 0.95 & 70 & 69.8 \\
\hline
\end{tabular}

Table 1. Comparison with the previously reported mathematical frameworks.

Once the validation purposes have been achieved, it becomes necessary to choose the devices structures to carry out the performance analysis. It is essential to inform at this point that the devices mentioned above were only adopted for validation purposes. The authors perceive the three devices structures obtained from [6] and [7] as potential candidates for RTD-based oscillators and the performance analysis will be performed on them. Afterward, it is quite interesting to verify how such devices depends on the applied bias voltage. Once again, RTD oscillator devices are voltage-controlled oscillator since its free-running frequency can be adjustable using the DC bias voltage. As previously discussed, the mathematical formalism proposed account for physical quantities which can also be treated as empirical parameters for fitting purposes. Only for convenience's sake, the I-V curves related to the fitting parameter will be omitted. Nonetheless, results presented a close agreement with the characterized from experimental measurements [6], [7]. Once the analysis of the full I-V characteristics has been laid out, it will move to investigate how the devices perform under bias conditions within the NDR. At first, it will be considered both the inductance and capacitance versus the DC voltage supply, as shown in Fig. 1. The idea is to make the proper choice of the inductance to resonate the tunnel diode capacitance at $\mathrm{THz}$ frequencies. Also, it will be possible to corroborate the capacitance values with the available in the related literature [6], [8], [9].

Initially, the results related to the Device 1 are shown in Fig. 1a. The reactive elements calculation is carried out for DC voltages supply from 9.7 to $10.5 \mathrm{~V}$. Such range of values are perpetuated from [6] and used here only for validation purposes. As one can see, the capacitance curve presents a maximum value to the order of $58.2 \mathrm{fF}$ at DC voltage supply value of $9.86 \mathrm{~V}$. This value proof the proposed formalism is robust once shows close agreement with [6]. From this, the proposed formalism can be employed to evaluate the inductance necessary to allow the resonant operation. As one can see, the inductance presents a minimum necessary (aligned with the peak capacitance) to guarantee the resonance activity to the order of $59.1 \mathrm{pH}$. Next, it is investigated both capacitance and inductance as a function of the DC voltage supply for the Device 2, as shown in Fig. 1b. In this case, results indicate a maximum capacitance to the order of $34.8 \mathrm{fF}$ at DC voltage supply value of $0.51 \mathrm{~V}$. Once again, the proposed formalism confirms to be accurate since such value is closed to the measured capacitance present from [8]. Further, the inductance necessary to compensate for the capacitive effect and bring the device to resonance is to the order of $1.1 \mathrm{pH}$. Finally, the proposed formalism is fully applied to determine both the values of the reactive elements related to RTD device presented in [9] and the results are shown in Fig 1c. Even though these values are not available in the literature, the authors of this manuscript consider such structure with potential application in oscillator circuits. In this case, the results indicated that the resonance occurs for capacitance and inductance to the order of $232.2 \mathrm{fF}$ and $121.4 \mathrm{pH}$, respectively, at DC voltage supply value of $0.311 \mathrm{~V}$.

With all devices' reactive parameters properly described, the merit figures associated with the RTD-based oscillator's performance can now be discussed. Remember that the merit figures adopted, namely oscillation frequency, maximum frequency and extinction ratio, are widely employed to perform analysis of oscillators. In addition, these results will give important clues on how efficient are the three devices into consideration. First is calculated the merit figures above mentioned as a function of DC voltage supply for the devices and the results are shown in Fig 2, with square, triangles, and circles symbols related to the maximum frequency, oscillation frequency, and extinction rate, respectively. Initially, the results for the device 1 is illustrated in Fig $2 \mathrm{a}$. As can be observed, both the maximum frequencies and the oscillation frequency to the order of $200.1 \mathrm{GHz}$ and $83.7 \mathrm{GHz}$, respectively, exhibit a near flat
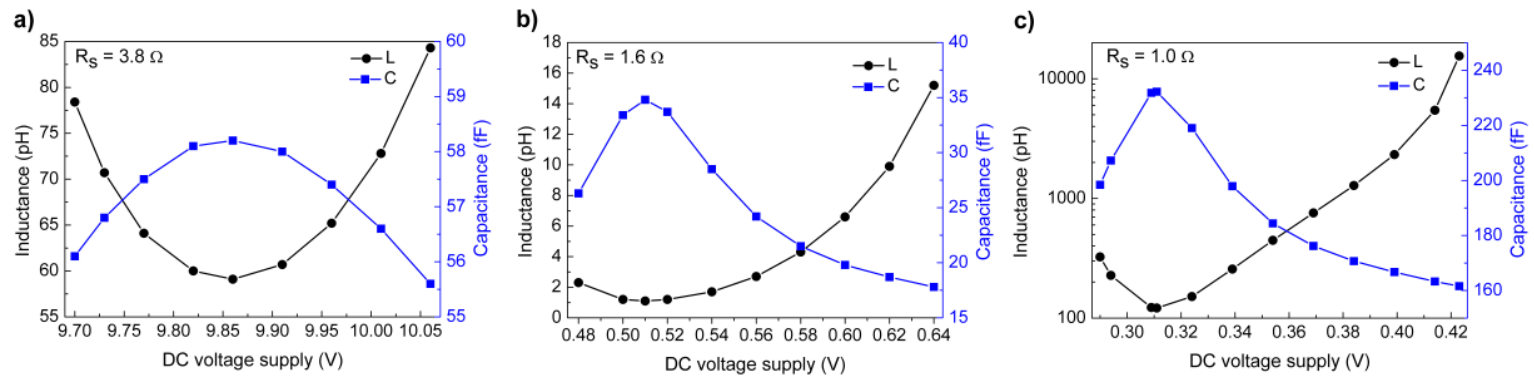

Figure 1. Capacitance and inductance values as a function of bias voltage for: (a) Device 1; (b) Device 2 and

(c) Device 3. The values of $\tau$ and $\tau c$ directly obtained from simulations are 1.314 ps and 0.388 ps (Device 1), 0.226 ps and $0.081 \mathrm{ps}$ (Device 2) and $6.747 \mathrm{ps}$ and $1.050 \mathrm{ps}$ (Device 3), respectively. 
dependence on DC voltage applied. This is particularly attractive because the oscillator can maintain the operating frequency even an undesirable variation occurs at such voltage. Moreover, the results present an extinction ratio value of $0.79 \mathrm{~dB}$ at DC voltage applied to the order of $9.86 \mathrm{~V}$.

Next, Fig $2 \mathrm{~b}$ illustrates the results for the device 2. In this case, the curves referring to the oscillatory characteristics of the device present a smooth region limited to the DC applied voltages from 0.50 to $0.52 \mathrm{~V}$. In this case, the results for the maximum frequencies and the oscillation frequency present values near to the order of $1350 \mathrm{GHz}$ and 760 $\mathrm{GHz}$, respectively. Even though such range coincides with the low value of $2.61 \mathrm{~dB}$ to the extinction ratio, this one is still considered acceptable in practical applications. Finally, the results related to device 3 are illustrate in Fig 1c. In contrast to the other devices, the maximum values of $295.4 \mathrm{GHz}$ and $157 \mathrm{GHz}$ for the maximum frequency and oscillation frequency, respectively, can only be achieved when the DC applied voltage are restricted to $0.31 \mathrm{~V}$. Additionally, the extinction ratio calculated presented the value of $6.32 \mathrm{~dB}$.

Therefore, these RTD-based oscillators are indeed potential candidates for Radio-over-Fiber (RoF) networks, where higher operation $\mathrm{THz}$ frequencies will be major network requirements.
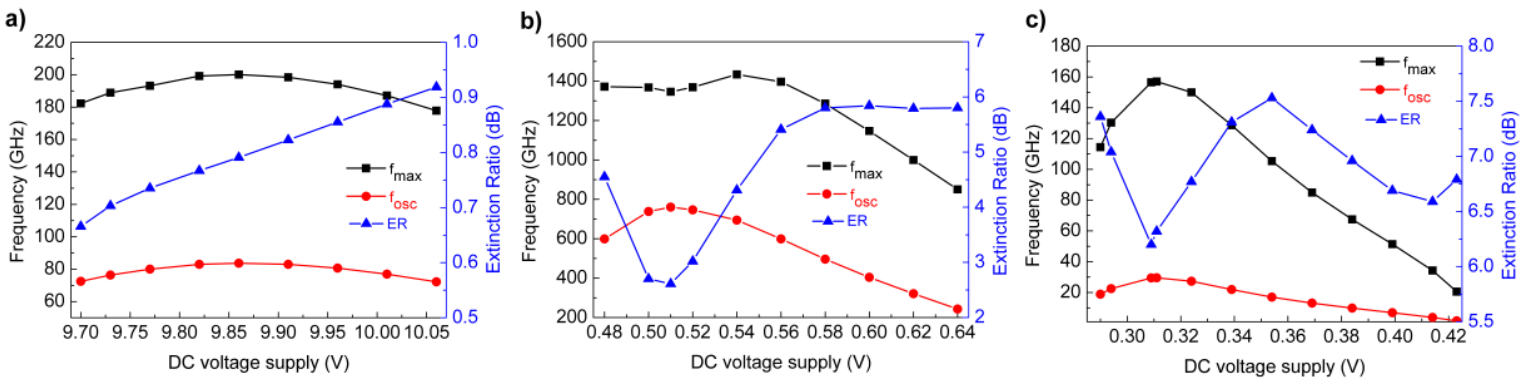

Figure 2. Oscillation frequency, maximum frequency and extinction ratio (ER) versus DC voltage supply for: (a)

Device 1; (b) Device 2 and; (c) Device 3.

\section{CONCLUSIONS}

In this paper, it is have carried out a comprehensive analysis of three configurations of oscillators circuits based on RTD. On top of that, it is investigated how each of these oscillators performs under DC bias applied within the NDR. In all cases, it is accounted for the DC bias effect over the capacitance and inductance of the reactive elements that composed the small signal model for oscillators. For this, a new expression for the width of the depletion region of the devices is derived. The proposed approach does not require the use of complicated methods to assess the performance of the RTD-based oscillators, which makes the performance analysis straightforward. Results indicated that all analyzed devices could stabilize very high-frequency oscillations as long as the DC bias applied is within a limited range of values within the NDR. Therefore, these devices are indeed potential candidates for RTD-based oscillators for future RoF networks scenarios, where higher frequencies to the order of $\mathrm{THz}$ will be major network requirements.

\section{ACKNOWLEDGEMENTS}

This work was supported by the Brazilian agency FAPESP.

\section{REFERENCES}

[1] J.N. Schulman, H.J. De Los Santos and D.H. Chow: Physics-based RTD current-voltage equation, IEEE Electron Device Letters, vol. 17, no. 5, pp. 220-222, May 1996.

[2] Q. Liu, et al.: Unified AC model for the resonant tunneling diode, IEEE Transactions on Electron Devices, vol. 51, no. 5, pp. 653-657, May 2004.

[3] H. Yamamoto, K. Miyamoto and T. Hayashi: Analytical Expression for the Tunneling Time in Symmetrical Rectangular Double-Barrier Structures, phys. stat. sol. (b), vol. 209, pp. 305-310, Jan. 1999.

[4] Y. Ando and T. Itoh: Calculation of transmission tunneling current across arbitrary potential barriers, Journal of Applied Physics, vol. 61, no. 4, pp. 1497-1502, Feb. 1987.

[5] L. Wang, et al.: DC characterization of tunnel diodes under stable non-oscillatory circuit conditions, IEEE Transactions on Electron Devices, vol. 58, no. 2, pp. 343-347, Feb. 2011.

[6] J. Encomendero et al:: Room temperature microwave oscillations in GaN/AlN resonant tunneling diodes with peak current densities up to $220 \mathrm{kA} / \mathrm{cm}^{2}$, Applied Physics Letters, vol. 112, 103101, Mar. 2018.

[7] T.C.L.G. Sollner et al:: Observation of millimeter-wave oscillations from resonant tunnelling diodes and some theoretical considerations of ultimate frequency limits, Applied Physics Letters, vol. 50, no. 6, pp. 332-334, Feb. 1987.

[8] S.G. Muttlak et al.: InGaAs/AlAs resonant tunneling diodes for THz applications: an experimental investigation, IEEE Journal of the Electron Devices Society, vol. 6, pp. 254-262, Feb. 2018.

[9] M.A.M. Zawawi and M. Missous: Design and fabrication of low power GaAs/AlAs resonant tunneling diodes, Solid-State Electronics, vol. 138, pp. 30-34, Dec. 2017. 